\title{
A definitive approach to rehabilitate acquired maxillary defect- A case report
}

\author{
Sreenivasulu .D ${ }^{1, *}$, Goverdhan .G' ${ }^{2}$, Himaja .K ${ }^{3}$ Chalpathi Rao .D ${ }^{4}$, Ravi Kumar .C ${ }^{5}$ \\ ${ }^{\mathbf{1}}$ Reader, ${ }^{\mathbf{2}}$ Post Graduate, ${ }^{\mathbf{3}} 3^{\text {rd }}$ Year Post Graduate, ${ }^{4}$ Professor, ${ }^{5}$ Professor and HOD, Dept. of Prosthodontics, Mamata Dental \\ College, Khammam, Telangana, India
}

*Corresponding Author:

Email: srivasmds@gmail.com

\begin{abstract}
Removal of tumors, carcinomas, trauma and congenital malformations results in acquired maxillary defects which greatly effect patients quality of life and self-esteem. Hemimaxillectomy patients mainly faces problem of mastication, phonetics and swallowing due to oro-antral communication. Prosthetic rehabilitation of hemimaxillectomy conditions can be challenging to prosthodontist and requires multidisciplinary approach for comprehensive care and treatment. Depending on size and location of the defect the amount of impairment and difficulty in prosthetic rehabilitation is seen. Hollow obturator prosthesis is most commonly used for rehabilitating hemimaxillectomy cases as an effective treatment. This case report discusses designing of cast partial denture and fabrication of hollow obturator prosthesis for above said condition to overcome problems like speech, swallowing, mastication and to improve quality of life.
\end{abstract}

Keywords: Hemimaxillectomy, Obturator, Prosthetic rehabilitation.

\section{Introduction}

Surgical resection of hard and soft palate defects due to acquired or congenital defects results in oroantral communication. This predispose the patient to hypernasal speech, fluid leakage into the nasal cavity, impaired masticatory function and psychological trauma. The main goal of maxillofacial prosthodontist is to close and separate the oral cavity from the sinonasal cavities to enhance clarity of speech, mastication, transport of bolus, avoidance of aspiration and nasal regurgitation. ${ }^{6}$

Obturator is a maxillofacial prosthesis used to close, cover, or maintain the integrity of the oral and nasal compartments resulting from a congenital, acquired, or developmental disease process, such as cancer, cleft palate, osteoradionecrosis of the palate; the prosthesis facilitates speech and deglutition by replacing those tissues lost because of the disease process and can, as a result, reduce nasal regurgitation and hypernasal speech, improve articulation, deglutition, and mastication. ${ }^{1}$

An obturator prosthesis is classified as surgical, interim, or definitive and reflects the intervention time period used in the maxillofacial rehabilitation of the patient; prosthodontic restoration of a defect often includes use of a surgical obturator, interim obturator, and definitive obturator. ${ }^{1}$ A definitive prosthesis is a more permanent prosthesis designed and fabricated to rule out the local recurrence when a surgical site healed and is dimensionally stable for a period between 6 months to one year. ${ }^{2}$

This case report discusses the prosthetic rehabilitation of hemimaxillectomy patient (armany class IV) with definitive cast partial hollow bulb obturator prosthesis to achieve optimum function.
Case Report

A 45 years old female patient has reported to the Department of prosthodontics, crown and bridge, Mamata Dental College and Hospital with a chief complaint of missing teeth, difficulty in chewing, drinking, speech and deformity of left side of face due to surgical removal of tumor. Her medical history was not contributory. The patient treatment report revealed that she was suffering from ameloblastoma of maxillary ridge extending from 12 to 27 regions, which was operated in department of oral and maxilla facial surgery, mamata dental college and hospital. On intra oral examination patient has armany class IV maxillectomy defect on left side of face associated with lack of lip support and depressed nasolabial fold. (Fig. 1) The patient was initially rehabilitated with interim obturator for 6 months and currently replaced with definitive cast partial hollow bulb obturator with replacing 12 to 27 teeth.

Maxillary and mandibular impressions were made using irreversible hydrocolloid impression material and casts were poured using die stones. These casts were surveyed and cast partial framework design was planned (Fig. 2) Embrasure clasps were planned in relation to 16,17 and 14,15 and 28 , cingulum rest and $i$ bar on 13. A complete palatal type major connector was planned. The mouth was prepared before making final impression using polyvinyl siloxane material and later master cast was poured. Blockouts were made before making the duplicate of the refractory cast. On this refractory cast desired wax pattern was fabricated and casting was carried out. Trial of this framework was done and needed adjustments were made. (Fig. 3A and B) Later occlusal rims were fabricated on framework and jaw relations were recorded. Trail of waxed up prosthesis with teeth was done. Once satisfactory 
esthetics and phonetics were achieved, the prosthesis was processed.

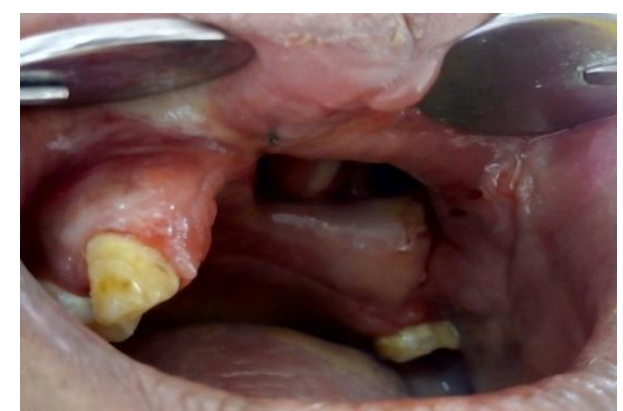

Fig. 1: Aramany class IV defect with slight modification

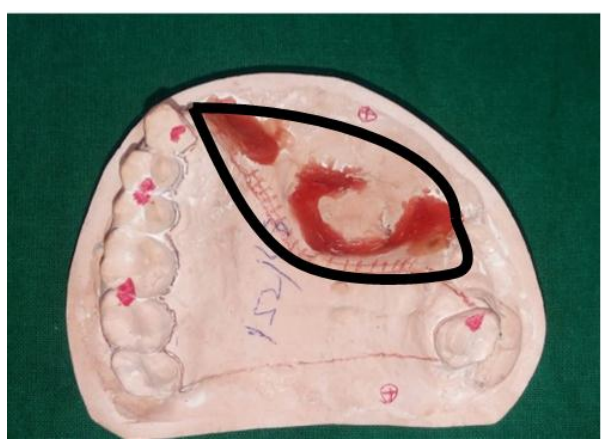

Fig. 2: Surveyed cast
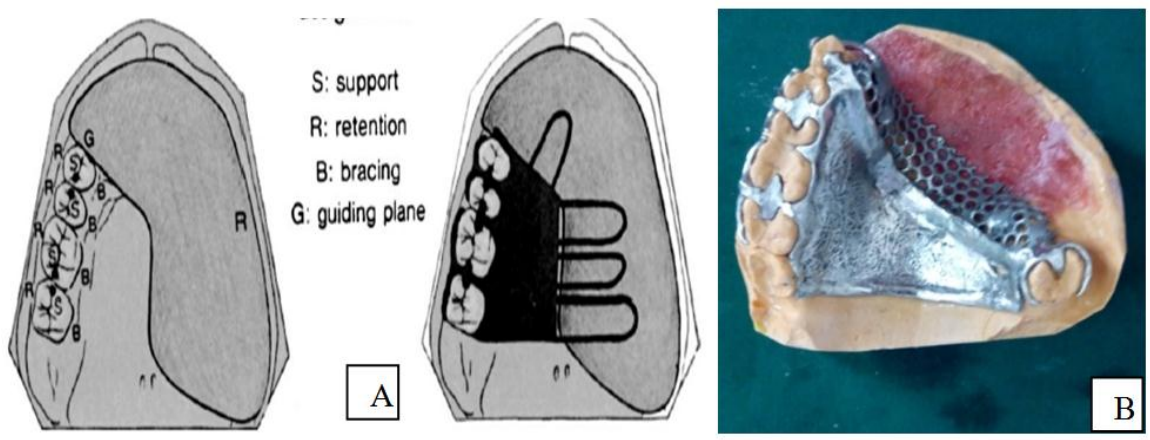

\section{Fig. 3: Metal frame-work try-in}

Hollowing of prosthesis was made to make denture light in weight. After dewaxing heat cure acrylic was packed on ridge area of the flask and some amount of resin was scooped out from the defect and was filled with sugar to make it hollow. The heat cure acrylic resin was packed in the remaining area of flask and curing was done. Later sugar was removed from processed denture by injecting hot water through hole and it was later covered by self cure acrylic resin. The final prosthesis was finished, polished and insertion was done. (Fig. 4A and B)
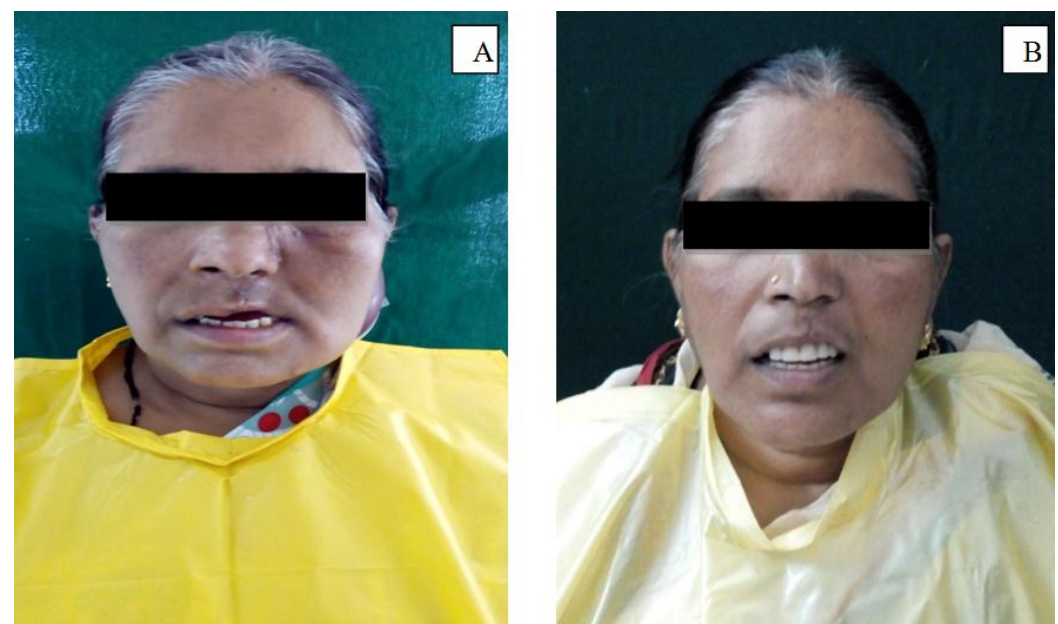

Fig 4: (A. Preoperative; (B Post operative after insertion of prosthesis

\section{Discussion}

Prosthetic rehabilitation using obturator helps to recover oral function in postsurgical maxillectomy defects. The patient presented here was planned for definitive prosthesis after stabilization and healing of defect following six months of surgery. It is also important to note that placement of obturator on unhealed tissues might lead to complications like pain, 
alterations in dimensional parameters, secondary infections etc. ${ }^{5}$

Cast partial denture designed in such a way that ibar casp placed adjacent to edentulous area and embrasure clasps on premolar and molars to enhance retention and bracing of denture. Rests are placed on teeth in order to increase support. The remaining palate and dentition is utilized to maximize the support, stability, and retention of an obturator bulb for successful functional prosthetic design restoration. ${ }^{7}$ Restablishment of architecture of nasolabial fold and prominence of lip support through wax build up during jaw relation paved way for esthetic enhancement.

Since weight of the obturator is one of the main disadvantages of the prosthesis, closed hollow bulb obturator is best choice for many patients as it is comfortable, maintains hygiene and most importantly light weight. Many investigators used different materials for hollowing out the prosthesis. Tanaka et al in 1977 used polyurethane foam material for hollowing out denture. ${ }^{3}$ In this present case we used sugar for hollowing out denture that would be efficient and economically viable.

\section{Conclusion}

Prosthetic rehabilitation with obturator is one of the best treatments for defects in maxillae arising from excision of tumors or any other conditions. Obturator helps to establish the structural and functional integrity. Present case has been rehabilitated with definitive cast partial hollow bulb obturator to enhance esthetics, speech and function which were lost due to surgical removal of ameloblastoma in addition to providing psychological gain.

\section{References}

1. The glossary of prosthodontic terms. J prosthet dent 2017;117(5s):e1-e105.

2. Curtis TA, Beumer J. Rehabilitation of acquired soft plate defects. In: Beumer J Curtis TA, Marunik MT, editors maxilla facial rehabilitation, prosthodontic and surgical considerations. $1^{\text {st }}$ edition st. Louis (MA): ishiyaku euroamerica; 1996. p.225-284.

3. Tanaka Y, Gold HO, Pruzansky. S. A simplified technique for fabricating a light weight obturator. $J$ Prosthet Dent 1977 Dec, 38(6):638-642.

4. Padmakar S. Patil, K. Rajkumar. Rehabilitation of hemimaxillectomy with foldable Obturator in Restricted Mouth Opening: A Case Report. J Indian Prosthodont Soc (Oct-Dec 2013) 13(4):612-616.

5. Filiz keyf, Obturator prosthesis for hemimaxillectomy patients. Journal of Oral Rehabilitation 2001,28;821-829.

6. Shruthi Jalan, Jogeswar Baram. Rehabilitation of hemimaxillectomy patient with definite hollow characterised obturator prosthesis. International journal of oral care and research 2016;4(3):216-219.

7. Gregory R. Parr et al. Prosthodontic principles in the framework design of maxillary obturator prostheses. $J$ Prosthet Dent 2005;93:405-11.

8. Dr. Lalit A Manek et al, Esthetics in Hemimaxillectomy: A Case Report. IOSR Journal of Dental and Medical Sciences 2017;16(5):1-4. 